\title{
Erratum: Synthetic magnetic fluxes and topological order in one-dimensional spin systems [Phys. Rev. A 91, 063612 (2015)]
}

Tobias Graß, Christine Muschik, Alessio Celi, Ravindra W. Chhajlany, and Maciej Lewenstein

(Received 3 July 2015; published 29 July 2015)

DOI: 10.1103/PhysRevA.92.019904

PACS number(s): 03.75.Be, 75.10.Pq, 03.65.Vf, 99.10.Cd

Our paper considers a spin chain with nearest-neighbor and next-nearest-neighbor interactions. To generate artificial magnetic fluxes, complex phases for every second nearest-neighbor interaction are chosen. The definition of these phases made in Fig. 2 of our paper is inconsistent with the performed numerical calculations. While Fig. 2 defined the phases on the horizontal links as $e^{i \pi \frac{p}{q}}, e^{2 i \pi \frac{p}{q}}, e^{3 i \pi \frac{p}{q}}$, all calculations were made with a different choice: $e^{i \pi \frac{p}{q}}, e^{3 i \pi \frac{p}{q}}, e^{5 i \pi \frac{p}{q}}$ (see the corrected version of Fig. 2 here). Accordingly, the phase definition in the main text should read $\varphi_{i+1, i}=\pi i(p / q) \delta_{(i+1) \bmod 2,0}$. With this redefinition, all results in the main text are correct as published, in particular the single-particle band structure shown in Figs. 3 and 4, the corresponding Chern numbers in Table I, and the many-body results presented in Fig. 5.

We note that the new definition doubles the flux per square plaquette $\Phi=2 \pi \frac{p}{q}$. The Harper-like equation (A1) in the Appendix of our paper has to be adapted accordingly. It should read

$$
H=-J \sum_{k, v}\left[e^{i\left(k+2 \pi \nu \frac{p}{q}\right)}\left(\sigma_{k \nu 1}^{+}, \sigma_{k \nu 2}^{+}\right)\left(\begin{array}{cc}
e^{i \theta} & 1 \\
0 & e^{i \theta}
\end{array}\right)\left(\begin{array}{c}
\sigma_{k \nu 1}^{-} \\
\sigma_{k \nu 2}^{-}
\end{array}\right)+\left(\sigma_{k \nu 1}^{+}, \sigma_{k \nu 2}^{+}\right)\left(\begin{array}{cc}
0 & 0 \\
e^{-i \pi \frac{p}{q}} & 0
\end{array}\right)\left(\begin{array}{l}
\sigma_{k(v+1) 1}^{-} \\
\sigma_{k(v+1) 2}^{-}
\end{array}\right)\right]+\text {H.c. }
$$

The wave number $k$ is defined in $[0,2 \pi / q]$. Therefore, $\sigma_{k v \ell}^{ \pm}=\sigma_{k(v+q) \ell}^{ \pm}$and $v$ takes values from 1 to $q$. As already stated in our paper, this leads to $2 q$ energy bands.

We thank Ralf Blattmann for making us aware of this inconsistency within our paper.
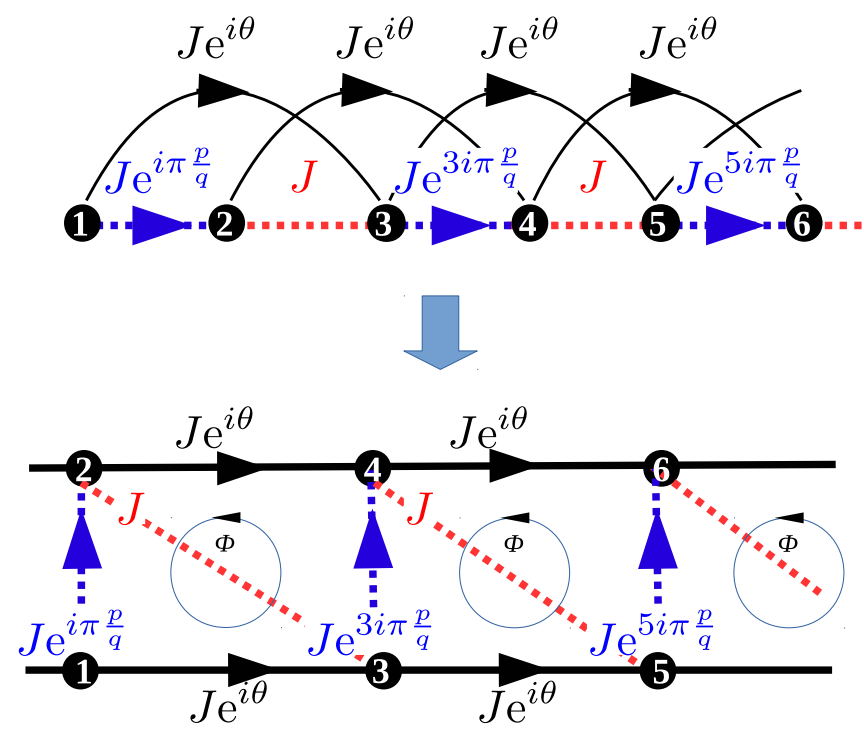

FIG. 2. (Color online) Corrected version of Fig. 2 in our paper. The phases on the blue bounds have been modified. 